\title{
Techniques for Technetium Scintigraphy in Plants
}

\author{
Geoffrey Currie ${ }^{1-3}$, Simon Clarke ${ }^{1,4}$, Suzy Rogiers ${ }^{1,4,5}$, and Janelle Wheat ${ }^{1,2}$ \\ ${ }^{1}$ Faculty of Science, Charles Sturt University, Wagga Wagga, New South Wales, Australia; ${ }^{2}$ Centre for Research in Complex Systems, \\ Charles Sturt University, Wagga Wagga, New South Wales, Australia; ${ }^{3}$ Australian School of Advanced Medicine, Macquarie University, \\ Sydney, New South Wales, Australia; ${ }^{4}$ National Wine and Grape Industry Centre, Charles Sturt University, Wagga Wagga, New South \\ Wales, Australia; and ${ }^{5}$ NSW Department of Primary Industries, Wagga Wagga, New South Wales, Australia
}

\begin{abstract}
The accumulation of technetium by plants has been widely studied and reported in the literature from the perspective of the incorporation of environmental ${ }^{99} \mathrm{Tc}$ into the food chain. Pertechnetate $\left(\mathrm{TcO}_{4}{ }^{-}\right)$is the most stable surface chemical form of technetium and is known to be extracted by plant roots, transported by the xylem, and reduced in the leaves; however, the mechanism of action is not entirely clear. Measuring the distribution of technetium in plants has been challenging, many questions remaining unanswered. To date, tracer studies for plant physiology (radionuclide and color dye) have relied on destructive sampling, prohibiting repeat-design experimentation. This article explores the technical issues relating to the application of scintigraphic imaging to plant physiology. The benefits and limitations of methods for introducing radiotracers to plants are outlined. Strategies for the successful labeling of various plant organs with ${ }^{99 \mathrm{~m} T c}$ and several unanticipated artifacts are described. The relevance of these labeling experiments to the study of plant vascular transport is explained, and strategies for optimizing the scintigraphic imaging of plants are outlined. Assessing plant physiology is an emerging frontier, especially given the growing importance of water management and the increased competing demand for crops as biofuels.
\end{abstract}

Key Words: instrumentation; vascular transport; pertechnetate; physiology; plant scintigraphy

J Nucl Med Technol 2010; 38:76-80

DOI: $10.2967 /$ jnmt.109.071886

\section{$\mathbf{T}$} he biodistribution of technetium in plants has been widely studied and reported in the literature. The first report of the use of pertechnetate as a tool for the study of plant vascular transport dates back to 1975 (1). The researchers were searching for a radiotracer that was mobile in the phloem, the vascular tissue responsible for transporting photosynthates from leaves to developing tissues. Their findings were inconclusive, and they published no further research on technetium after establishing methods for detecting phloem transport of ${ }^{11} \mathrm{C}$ fed to leaves as carbon

\footnotetext{
Received Oct. 23, 2009; revision accepted Mar. 26, 2010.

For correspondence or reprints contact: Geoffrey Currie, Faculty of Science, Locked Bag 588, Charles Sturt University, Wagga Wagga 2678, New South Wales, Australia.

E-mail: gcurrie@csu.edu.au

COPYRIGHT @ 2010 by the Society of Nuclear Medicine, Inc.
}

dioxide (2). Subsequent research involving technetium and plants focused on the incorporation of environmental ${ }^{99} \mathrm{Tc}$ into the food chain via plants grown in soils polluted by nuclear accidents and nuclear weapons testing and detonation $(3,4)$.

Pertechnetate $\left(\mathrm{TcO}_{4}{ }^{-}\right)$is the most stable surface chemical form of technetium and has been reported to be highly mobile in biogeochemical cycles (5). $\mathrm{TcO}_{4}{ }^{-}$is known to be extracted by plant roots, transported by the xylem, and reduced in the leaves because technetium is not evaporated (5). The xylem is the vascular tissue primarily responsible for transporting sap from roots to transpiring organs, such as leaves. In 1999, the role of scintigraphy in the nondestructive quantification of radiotracers in plants was first described (4). The paucity of research since then probably reflects the lack of access of plant scientists to scintigraphic equipment and associated expertise, the unfamiliarity of plant physiologists with the imaging capabilities of nuclear medicine, and the limited exposure of nuclear medicine practitioners to research in plant sciences.

The main purpose of this article is to inform nuclear medicine and plant scientists of imaging methods applicable to the study of plant vascular transport by describing the scintigraphic detection of metastable ${ }^{99} \mathrm{Tc}\left({ }^{99 \mathrm{~m}} \mathrm{Tc}\right)$. We refer primarily to technetium in the form of $99 \mathrm{~m} \mathrm{Tc}$ pertechnetate $\left({ }^{99} \mathrm{mcO}_{4}{ }^{-}\right)$, but our research is relevant to any $\gamma$-emitting radionuclide that is mobile in the xylem vessels of plants.

\section{BENEFITS OF SCINTIGRAPHY}

To date, tracer studies for plant physiology (radionuclide label or color dye) have relied on destructive plant sampling. That is, an individual plant (or part thereof) is sacrificed and dissected at a specific time interval for tracer analysis. Consequently, that same plant cannot be used to provide time series or dynamic data and cannot be used in repeat testing. Scintigraphy has several valuable advantages over existing techniques:

- Sampling is nondestructive; therefore, the plant remains intact for repeat-design experimentation.

- A single plant can be used to provide contiguous data over time, both reducing the number of plants required 
and significantly increasing the sampling rate (and thus the accuracy of parameter estimation).

- Sampling can readily begin at time zero (i.e., application of the radiotracer), offering a broad window of continuous data sampling.

- Dynamic data permit the quantitation of tracer movement on the basis of the region of interest (ROI), improving both the accuracy and the reproducibility of results, especially over those of visual assessment alone.

- Reductions in costs are achieved through reductions in the number of plants required and tracer quantity. Further reductions in costs are achieved through comparative streamlining of data collection and analysis and reductions in manual sampling and dissection.

In brief, scintigraphy provides significantly improved data quality in a more efficient and reproducible manner for the exploration of time-related changes in plants.

\section{METHODS OF TRACER INTRODUCTION}

The approaches described here are not intended to be exhaustive, as numerous other approaches are possible. The specific method chosen depends on the physiologic process under investigation.

A simple, nondestructive means of introducing a radionuclide to a plant is through root uptake. For hydroponically grown plants, this process is as straightforward as introducing the radionuclide to the hydroponic solution. Unfortunately, radionuclides such as ${ }^{99 \mathrm{~m}} \mathrm{Tc}$ (as pertechnetate) tend to bind with nutrients in the solution, leading to less efficient root uptake. Replacing (after a washing process) the hydroponic solution with tap water improves radionuclide uptake by the root system but only marginally. Distilled (deionized) water is the ideal medium in which to house roots for radionuclide introduction. The same technique can be used for potted plants after the roots are removed from the soil and washed. Care must be taken when transferring and washing the roots because uptake and transport processes can be changed by damage to the roots (microscopic damage to root hairs is sufficient) or air emboli resulting from even brief periods out of solution. The roots should be housed in a container with a volume as small as possible to improve the specific concentration.

For plants grown in potted soil, a radionuclide solution can be added directly to the soil. However, precautions must be taken when applying a radionuclide solution to soil. The radionuclide should be contained in a volume that allows sufficient dilution and diffuse distribution within the soil. Conversely, the specific concentration must be sufficiently high to allow measurable quantities to be taken up by the root system. Generally, this parameter requires the use of a radiation dose much higher than that required for the distilled water method outlined earlier; in turn, a high radiation dose raises concerns about radiation safety and image degradation due to radiation scattered into the field of view of the imaging system. The latter can be managed by ensuring that the soil surface is a sufficient distance from the imaging field of view, washing the solution after surface application or injecting the solution below the surface layer of the soil $(>5 \mathrm{~cm})$, and using lead shielding around the soil surface.

Inserting multiple watering spikes into the soil can facilitate improved delivery of the radionuclide more directly to the roots while reducing the risk of contamination, reduce the dilution volume, and improve the efficiency of root uptake. However, this method is inefficient in comparison with methods without soil (described earlier) because variations in radionuclide labeling of soil components decreases root uptake. The level of hydration of the soil (and plant) also has a significant bearing on the efficiency with which a soil-based radionuclide is absorbed. Well-hydrated soils result in slower uptake by the plant, whereas poorly hydrated soils can result in trapping of the radionuclide in the soil solution or rapid physical transit through the soil to the bottom of the pot. Different soil types absorb in different ways; therefore, the efficiency of uptake of radionuclides from soil by roots can vary with soil type. For example, technetium extraction is inhibited in anaerobic soils, particularly those with high concentrations of organic matter, because of the predominance of $\mathrm{TcO}_{2}$ as opposed to the more familiar form, $\mathrm{TcO}_{4}(3)$.

The uptake of a radionuclide by roots is a time-consuming process and results in dilute quantities of the radionuclide in plant structures, particularly in samples from soil. Circumventing delays in root uptake can be achieved with the "cut-shoot" method, in which the shoot of interest is simply cut from the remainder of the plant and introduced to a solution of a radionuclide in distilled water. Care must be taken to avoid the introduction of air into the xylem vessel by, under ideal conditions, cutting the shoot while it is submerged. Failing this, the shoot should be placed in the solution immediately after being cut. Another drawback of this approach is that the excision might result in altered physiology proximal to the cutting site. Generally, this method is considered destructive sampling, prohibiting repeat-design experimentation, although it might provide dynamic data. The principal advantage of this method is that it allows rapid accumulation into plant organs (Fig. 1) and thereby effective evaluation of gross physiology (e.g., relative accumulation in leaves or fruit).

An alternative approach for labeling plant tissues is the "wick" method. In brief, a wetted cotton thread is pulled through a plant organ with a needle (e.g., fruit or stem). One end of the thread is placed in a sealed reservoir of a concentrated radionuclide, and the other end is exposed to air. As evaporation dries the cotton thread, the radionuclide is drawn up the thread and into the plant organ. Care must be taken to prevent the cotton thread from touching any plant structure and risking contamination. The obvious advantage of this approach is that the radionuclide can be 


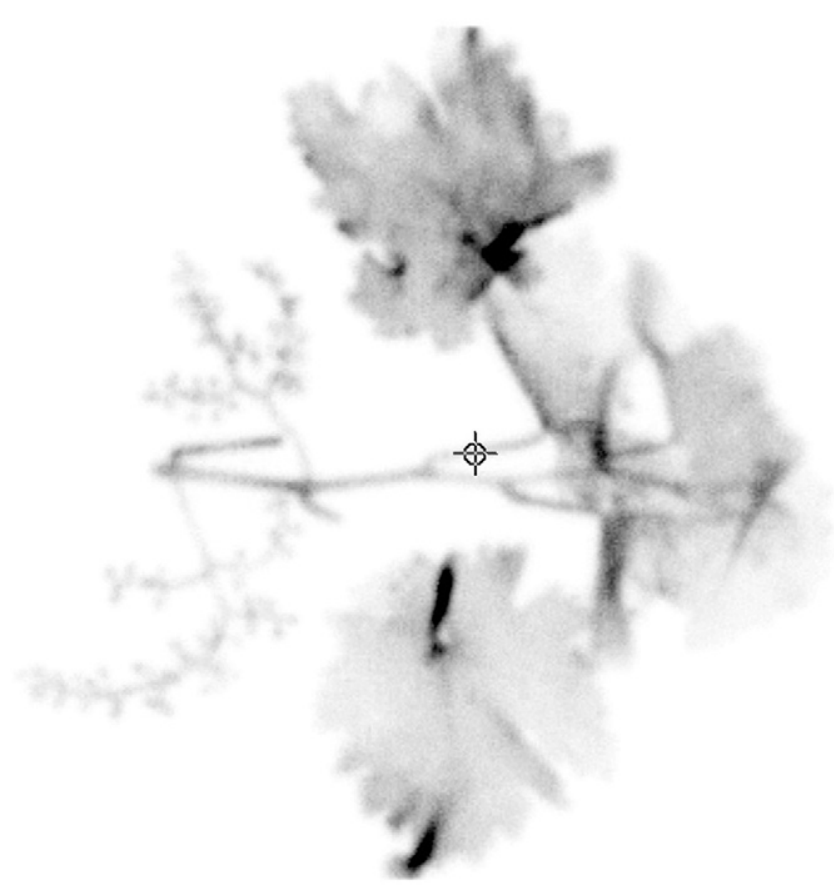

FIGURE 1. Cut-shoot method allows rapid accumulation of radionuclide in plant structures. Scintigraphy of cut shoot reveals accumulation in stem and leaves. Note absence of accumulation in fruit itself. ${ }^{99 \mathrm{mT}} \mathrm{T}$-pertechnetate accumulates in unripe fruit but not in ripe fruit.

introduced at specific locations in the plant. The major disadvantage of this method is that the rates of transport and dilution of the concentrated radionuclide within the plant depend on the rate at which the radionuclide is loaded into the xylem. Therefore, a highly concentrated (highspecific-activity) radionuclide is recommended. Another disadvantage of this method is that it ruptures xylem vessels, in turn facilitating the movement of sap away from the wick site. Additionally, the focal increase in the accumulation of the radionuclide at the wick site may impede the evaluation of proximal structures, particularly if transport rates are low. Nonetheless, the method has allowed the demonstration of bidirectional ${ }^{99 \mathrm{~m}} \mathrm{Tc}$-pertechnetate movement away from the wick site (Fig. 2).

In foliar application, a radionuclide solution is applied directly to the leaves. In this approach, contamination is a major concern, requiring meticulous attention to technique. Reverse xylem transport must be encouraged by inhibiting transpiration from the labeled leaves. This process is adequately achieved by wrapping the labeled leaves or stem in aluminum foil and encouraging transpiration from the nonlabeled leaves. Unfortunately, radionuclide transport is usually slow because the volume and concentration of radiolabeled water are low. Nonetheless, this technique has been used to successfully demonstrate the movement of ${ }^{99 \mathrm{~m}} \mathrm{Tc}$-pertechnetate from the leaf back to the plant stem; this finding discounts the previous understanding that ${ }^{99 \mathrm{~m}} \mathrm{Tc}-$ pertechnetate is irreversibly reduced in leaves (4) and indicates movement in a proximal direction (Fig. 3).

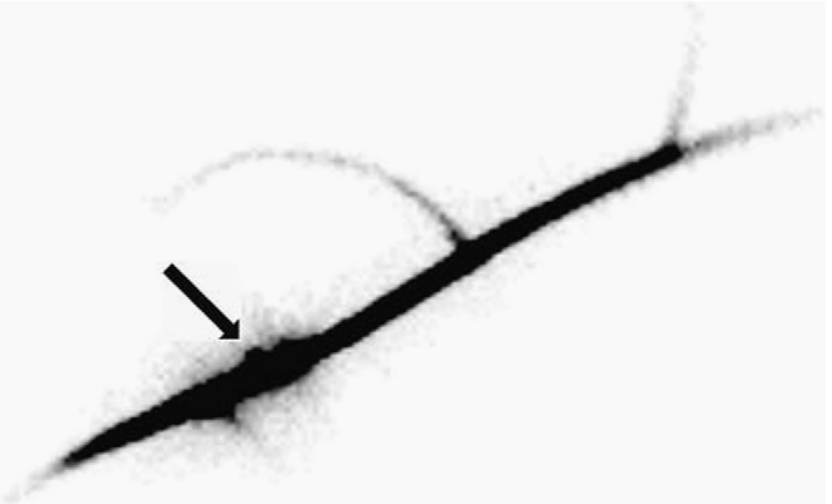

FIGURE 2. Wick method demonstrates bidirectional movement of tracer from wick site (arrow). Distal part of grape stem is at top right of image, and proximal (backflow) part of stem is at bottom left of image.

Immersing specific organs in a radionuclide solution is another labeling method, but it has significant contamination risks. Plant organs such as leaves, fruit, or an individual root can be immersed in a reservoir containing a radionuclide solution to provide continual exposure to the radioactive solution, leading to uptake and transport of the radionuclide. This method provides better results than foliar application but may not represent true physiologic conditions. An example of this approach that has been found to be useful is the introduction of a radionuclide via a single root. This technique is minimally invasive and allows the functional evaluation of a specific pathway. In this example, a window is cut into the side of a plastic pot containing a plant (Fig. 4). A single root is "dug" out through the window, taking care to minimize damage to the roots. The root is immersed in a container of radionuclide solution and the container is wrapped in foil to eliminate light exposure. It has been observed that individual roots service specific branches of grapevine plants.

\section{TECHNICAL CONSIDERATIONS}

Consideration should be given to the use of image merge software, with which a scintigraphic image can be referenced against a photograph for localization and analysis. Traditionally, anatomic localization might be undertaken with a transmission scan to create a silhouette or shadow of the anatomic structure. The photographic fusion approach overcomes the possible limitations of using transmission scanning of very thin and low-density objects and provides a significant improvement in the ability to accurately localize the distribution of activity within a structure. Numerous research studies performed in nuclear medicine departments might be assisted by fusion of planar images with photographs. This technique might be particularly useful, as is the case with plant physiology, if the research involves specialists from outside the nuclear medicine domain. Nonetheless, consideration must be given to alignment errors associated with the merging of an image acquired in a planar fashion 


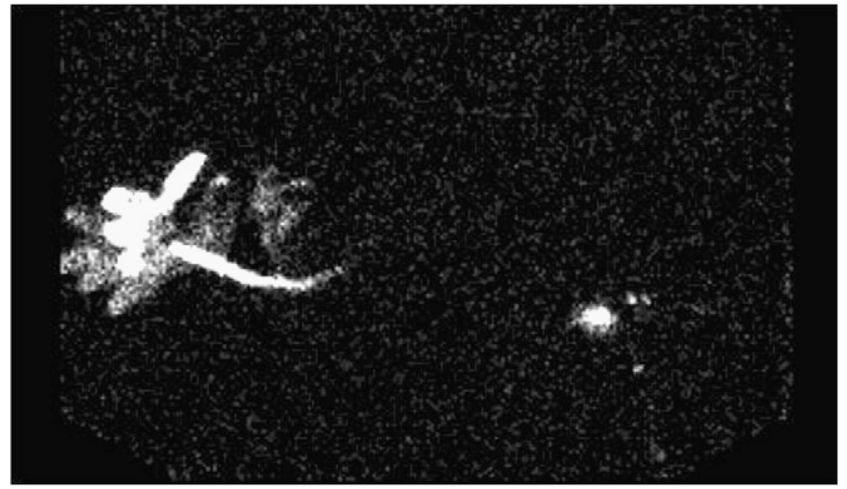

FIGURE 3. Activity applied to leaf (left) migrates down stem. Activity is concentrated at junction of main and branch stems (right).

(scintigraphy) with an image acquired in a pinhole fashion (photography), particularly toward the periphery of the image (Fig. 4). These difficulties can be overcome with software distortion or simply by maximizing the object-toaperture distance for the photograph.

The environment in which a standard $\gamma$-camera is housed (temperature and lighting) tends to be less than ideal for plant physiology. The $\gamma$-camera environment must be modified to induce transpiration; modifications might include artificial lighting, higher temperatures, and fans for air movement. The availability of natural light through windows is an advantage. Plant positioning is also important because movement and too much distance from the object to the detector can reduce image quality and resolution. Transpiration from individual leaves can be reduced or inhibited if the leaves are positioned too close to the detector or by mechanisms designed to secure the position of the plant. In particular, leaves should not be pressed flat or taped against the surface of the camera during the collection of dynamic data.

Acquisition parameters include the use of an "upright" gantry configuration to image plants vertically. Plants can be placed in a horizontal position for SPECT. Dynamic acquisition at 1 frame per 5-10 $\min$ for $3 \mathrm{~h}$ and then 1 frame per $30 \mathrm{~min}$ up to $24 \mathrm{~h}$ is generally used with a 256 matrix. The photopeak should be $15 \%$ centered on $144 \mathrm{keV}$ to eliminate scatter events (6).

One should aim for $100 \mathrm{MBq}(2.7 \mathrm{mCi})$ in $100 \mathrm{~mL}$ of solution and a total dose of 400-500 MBq (10.8-13.5 mCi) if roots are to be submersed in water and for foliar applications. Four doses of $100 \mathrm{MBq}(2.7 \mathrm{mCi})$ in $10 \mathrm{~mL}$ of solution are best for direct administration to roots from soil (with the aid of several watering spikes), and a dose of 100 $\mathrm{MBq}(2.7 \mathrm{mCi})$ per milliliter is appropriate for the wick method. In all cases, the uptake period depends on the transpiration rate and soil hydration; however, as a general rule, 2-4 h of accumulation should be used for physiologic uptake, and $30 \mathrm{~min}$ should be used for the cut-shoot application. Sodium chloride tends to be toxic to many

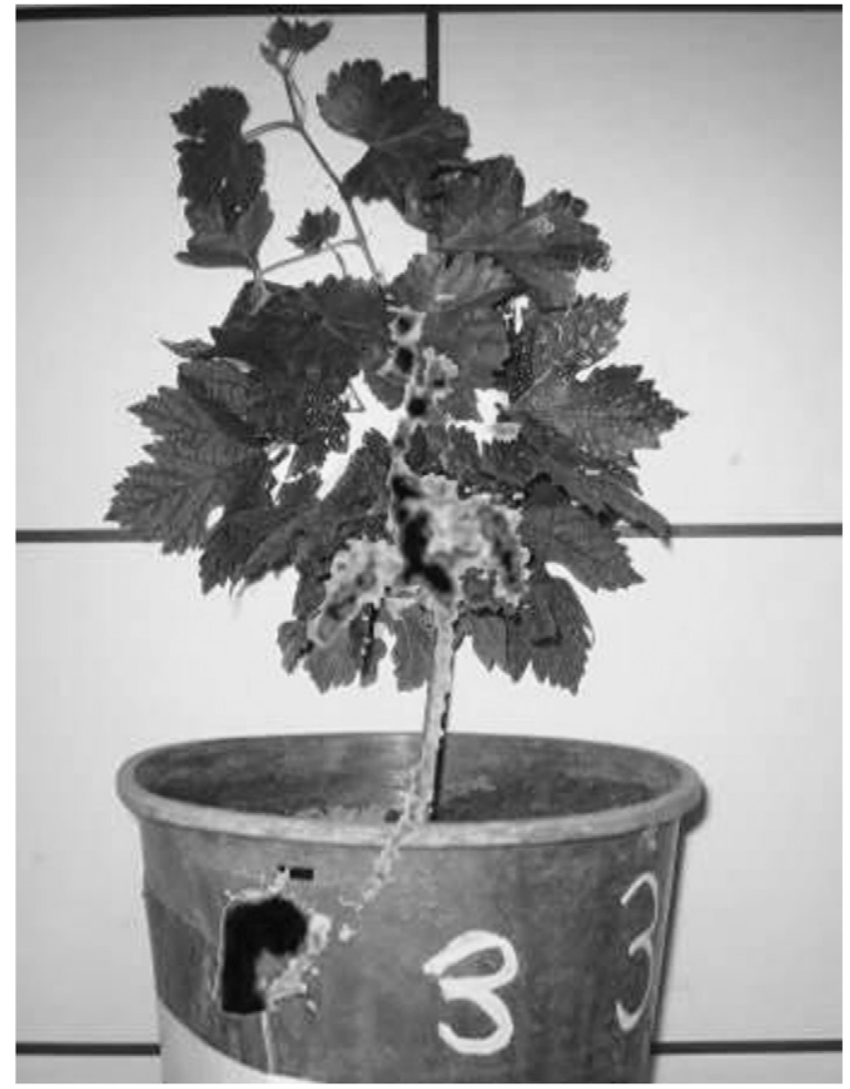

FIGURE 4. Merging of photographic and scintigraphic image data for improved localization. Under ideal circumstances, display would be in color.

plants, albeit at high concentrations; therefore, radionuclide doses should be diluted with deionized water.

Plant imaging presents unique radiation safety issues because the bulk of the radiation dose is not subject to large amounts of attenuation, which might be encountered for internalized human doses. Time, distance, and shielding are particularly important principles to apply during the uptake phase, when a large component of the dose for each plant resides in external containers. Deep soil administration provides some degree of shielding, but the storage of a large volume of plants requires dedicated shielding or an isolated room that still provides an environment conducive to transpiration. The use of radiation in an open delivery system (e.g., spray bottle or open container) involves a substantial contamination risk and requires the use of gloves, gowns, shoe covers, and a generous application of absorbent sheets.

\section{CONCLUSION}

We have demonstrated that the scintigraphic detection of ${ }^{99 \mathrm{~m}} \mathrm{Tc}$ is a powerful tool for the study of xylem transport because ${ }^{99} \mathrm{mcO}_{4}{ }^{-}$behaves as a xylem nutrient analog and the distribution of the radionuclide is amenable to nondestructive visualization and quantitation. Nuclear medicine imaging is a well-established research tool and has 
broad applications in both research and clinical studies of human and animal populations. Assessing plant physiology with the techniques described in this article represents an emerging frontier for nuclear medicine and quantitative plant physiology and is likely to play an important role in agricultural water management given the emerging demand for biofuels.

\section{REFERENCES}

1. Pickard WF, Hill RL. An application of gamma-scintigraphy to the observation of basipetal transport in moonflower. Planta. 1975;125:289-301.
2. Pickard WF, Minchin PEH, Troughton JH. Real time studies of carbon-11 translocation in moonflower: the effects of cold blocks. J Exp Bot. 1978;29:993-1001.

3. Bennett R, Willey N. Soil availability, plant uptake and soil to plant transfer of ${ }^{99}$ Tc: a review. J Environ Radioact. 2003;65:215-231.

4. Krijger GC, Verburg TG, Den Hollander W, Hermans PMJA, De Goeij JJM, Wolterbeek H. Approaches for nondestructive spatial distribution measurements in whole plants using radionuclides. J Plant Physiol. 1999;155:165-172.

5. Tagami K, Uchida S. Comparison of transfer and distribution of technetium and rhenium in radish plants from nutrient solution. Appl Radiat Isot. 2004;61:12031210.

6. Currie GM, Wheat JM. Small angle gamma ray scatter: what is the impact on image quality? Internet J Med Technol. 2008;4. Available at: http://www.ispub. com/journal/the_internet_journal_of_medical_technology/volume_4_number_ 2_51/article/small_angle_gamma_ray_scatter_what_is_the_impact_on_image_ quality.html. Accessed April 12, 2010. 